\title{
Estimating diffusion and price elasticity with application to telecommunications
}

\author{
Christos Michalakelis • Georgia Dede • \\ Dimitris Varoutas • Thomas Sphicopoulos
}

Accepted: 23 November 2010 / Published online: 21 December 2010

(C) Springer Science+Business Media, LLC 2010

\begin{abstract}
This paper aims to propose a new framework for estimating and forecasting diffusion of high technology products, along with the construction of a price index. Into that context, the "diffusion-price" model is presented, as an innovative concept providing a long term estimation of both price and diffusion elasticity. This corresponds to the bidirectional estimation of the mutual influence of the product's price over its expected diffusion and vice versa. The discrete parts of the methodology are the use of a diffusion model for the initial estimation of diffusion, the construction of a price index function for estimating the pricing mechanism and, finally, the construction of the "diffusion-price" model for estimating and adjusting the diffusion level and price quantities. The case studies examined, whose solution was based on genetic algorithms, revealed remarkable results which can be used for business strategies development, as the pricing policy is able to make diffusion diverge substantially from the initial estimates. The case studies considered correspond to the ADSL technology diffusion in the wider European area.
\end{abstract}

C. Michalakelis $(\bowtie) \cdot$ G. Dede $\cdot$ D. Varoutas · T. Sphicopoulos

Department of Informatics and Telecommunications,

National and Kapodistrian University of Athens,

Panepistimiopolis, Ilisia, Athens 15784, Greece

e-mail:michalak@di.uoa.gr

D. Varoutas

e-mail: arkas@di.uoa.gr

T. Sphicopoulos

e-mail: thomas@di.uoa.gr

G. Dede

Department of Informatics and Telematics, Harokopio University of Athens,

70, El. Venizelou Str, Kallithea, Athens 17671, Greece

e-mail: gdede@di.uoa.gr 
Keywords Diffusion • Forecasting • Price indices • Diffusion-price model •

Diffusion elasticity $\cdot$ Price elasticity

\section{Introduction}

The telecommunications sector is a high-tech area which corresponds to one of the most significant contemporary investments. It is strongly related with rapid changes occurring more frequently than in any other sector. Moreover, privatization and deregulation of the telecom market, together with the effects of increasing competition and the introduction of new services, resulted in the emergence of new problems regarding technology diffusion forecasting, under a high level of uncertainty and a need for risk management, mainly due to competition.

Modeling and forecasting the diffusion of innovations has attracted the interest of practical and academic studies since some decades ago, initiated by the pioneering works of [1-3], and others. The interest for the estimation and forecasting of innovations diffusion patterns is now extended to all kinds of high technology markets. The corresponding literature includes some important contributions, such as [4] where general diffusion patterns for information technology innovations are suggested, [5] where a thorough overview, together with a method for planning and implementing forecasts for disruptive and discontinuous innovations are presented and [6] containing an informative review regarding the research conducted towards diffusion modeling.

The importance of the above considerations becomes more crucial if the fact that telecommunications are almost always connected to heavy investments and critical business plans is taken into account. Industrial plans rolled out in an attempt to meet market's demand and growing competition must be precisely forecasted, in terms of the expected level of adoption and the expected market shares, together with the revenue consequences for both new and established products. Failure to produce reasonably accurate forecasts will most probably lead to dramatic sequences to corresponding supply, e.g. oversupply and unneeded over-investments, or under-utilization of a firm's capacities.

Moreover, the importance of innovations diffusion estimation and forecasting is extended to all kinds of high technology markets as discussed in [4] where general diffusion patterns are suggested for information technology innovations, in [5] where an thorough literature overview is presented and a method for planning and implementing forecasts for disruptive and discontinuous innovations is proposed and in [7] where a review of telecommunications demand forecasting is presented.

Although forecasting models for established products and services are well developed, new opportunities have emerged due to the nature of the high technology products' market. Therefore, further methodological work should be carried out, by identifying the gaps that have opened up, due to the change of the markets' scope and structure. The present work targets exactly to this 
point; to make the diffusion estimation process more accurate and provide indications for a proper pricing policy.

The question of acceptance or rejection of a product is a quite complicated aspect which affected by a number of decision variables. The most determinative ones are the market structure from the standpoint of competition, the product's marketing strategy and, of course, its price. Marketing strategy and pricing policies influence the main characteristics of a product's life cycle, which are the market potential and the period of its existence. Carefully planned marketing strategies, such as product bundling or tying can sustain its life time as well as its initially estimated saturation level.

A product's price is among the most important factors that determine its life cycle, from the early stages until market saturation. Usually, most of the technological innovations are introduced immediately after this is technically feasible, however at relative high prices. This discourages the potential adopters from accepting these products at this early stage, even if they may correspond to radical technological breakthroughs. However, this situation is usually reverted when price reductions occur, which make adoption affordable. Intuitively, the diffusion process can be accelerated if suitable pricing policies are adopted which will in turn lead to price reduction since, the more the adopters the easier for the supplier to set prices closer to the product's marginal cost of production.

This last consideration constitutes the main contribution of the present work, as it describes the way diffusion and price interact, resulting into the adjustment of the initial estimations for both quantities. The proposed methodology bridges the currently existing gap in diffusion theory, where the corresponding models consider mainly the time as the only diffusion variable, into which the underlying diffusion dynamics are accommodated.

Some important work towards this direction is presented in [8] where the problem is addressed and analyzed by the supplier's point of view. Towards this direction, market demand and costs are incorporated into a dynamic pricing model, in order to enhance the long run profit performance. On the other hand, the present work is devoted to establishing a methodology that captures the simultaneous cause-effect relationship between diffusion and price, taking into account the consumer's view of the market. To accomplish this, a price model is developed, built upon a product's main characteristics, according to the perceived utility of the consumers. Using this price model and the estimated demand, the proposed methodology is used for the estimation of the interaction and cross-elasticity between price and demand.

Similar approaches, regarding pricing schemes in the market of high technology can be found in [9], where an alternative criterion for determining the Internet settlement between Internet Service Providers (ISPs) is presented. According to the demonstration, this rule makes ISPs easier to make a peering arrangement. In this model, the traffic volume is a function of a market share. In addition, in [10] a communication network, used by customers with heterogeneous service requirements is analyzed, based on the assumption that there is an infinite population of customers, who join the network as long as 
their utility is larger than the price of the service. For both models considered in this work the prices that maximize the provider's profit are determined by using elements from queuing theory, as well as from microeconomics and game theory. It is worth mentioning that in [11] an overview of tariffs for Asymmetric Digital Subscriber Line (ADSL) connections across Europe is presented and a hedonic model is applied in order to identify and estimate the influence characteristics of the broadband prices' shape and a price index for ADSL connections is constructed. Finally, in [12] a hedonic study on mobile telephony market in France is presented, together with pricing-quality strategies, whereas in [13] a game with competition among providers is constructed, in order to understand consumers' preferences and define the most profitable bundles, with their associated prices.

An important thing needed to be addressed is that the diffusion process of a high technology product is also influenced by factors located outside the referenced market. The most characteristic of them is the influential pressure of the adoption level of the neighboring countries or of the adjacent markets, in general [14].

The present work is formed as follows: Section 2 presents the basic concepts of the theoretical background of diffusion theory and models and hedonic functions (price indices) construction. Section 3 is devoted to the development of the introduced methodology, together with a brief overview of genetic algorithms, which are used to solve the necessary mathematical formulations. Section 4 presents a number of case studies for the diffusion and pricing of the ADSL technology in some European countries, as well as the analysis of the results. Finally, Section 5 provides some useful conclusions and directions for future research.

\section{Diffusions models and hedonic functions}

\subsection{Diffusion models}

Diffusion models are mathematical functions of time, used to estimate the parameters of the diffusion process of a product's life cycle, usually at an aggregate level, without taking into consideration the underlying specific parameters that drive the process. The most well-known representatives of the models developed for diffusion estimation, are the Bass model [1], the FisherPry model [15], the Logistic family models [16], as well as the Gompertz model [17].

Logistic models and variations of the Gompertz model produce $S$-shaped curves, which are commonly used for forecasting the diffusion of products or services at the aggregate level, which is the total market response, rather than at the individual customer level. $S$-shaped patterns derive from the differential equation described below:

$$
\frac{d Y(t)}{d t}=\delta \cdot Y(t) \cdot[S-Y(t)]
$$


In Eq. $1, Y(t)$ represents the total penetration until time $t, S$ is the saturation level of the specific technology and $\delta$ is a constant of proportionality, called coefficient of diffusion. Penetration is defined as the proportion of the population that adopts the product or the service examined.

At the time that the particular technology is introduced $(t=0)$, there is a critical mass, the "innovators" that initially adopt it. This number influences the rate of diffusion until the time market reaches its saturation level. The other main group of adopters that exists, apart from the innovators, corresponds to the imitators. These proceed to adopting the innovation as a consequence of influence from the innovators and the pressure of the social system, such as advertising and the word-of-mouth.

The family of diffusion models described here is widely used in order to estimate a diffusion process, regarding its potential and its shape. In the area of telecommunications, these models were used in [18], in order to study the diffusion of mobile telephony services.

In the context of this work, the Linear Logistic model is used in order to estimate the penetration of the technology at each year. The general form of the Logistic models is described by the following equation:

$$
Y(t)=\frac{S}{1+e^{f(t)}}
$$

where $Y(t)$ is the estimated diffusion level and $S$ is the saturation level, while $f(t)$ is given by the following formula:

$$
f(t)=-a-b \cdot t(m, k)
$$

$t(m, k)$ is a function of time, which is estimated through one of the following formulations, according to the model used:

1. Linear:

$$
t(m, k)=t
$$

2. Box-Cox:

$$
t(m, k)=\frac{\left[(1+t)^{m}-1\right]}{m}, k=0
$$

3. Flog:

$$
Y(t)=\frac{\left\{\left[(1+k t)^{\frac{1}{k}}\right]^{m}-1\right\}}{m}
$$

4. Tonic:

$$
Y(t)=\frac{S}{\left(1+e^{a+b t}\right)^{c}}
$$

As far as the present work is concerned, the Linear instance of the model, also known as the Fisher-Pry model [15], is considered for the development of the proposed methodology. 
As far as the study of the impact of decision variables into the diffusion process is concerned some important work has already been introduced in literature $[19,20]$. However, the added value of the present work is that the decision variable participates into the constructed model not simply as a recorded value, but as an output of a function based on the corresponding theory of price indices and hedonic functions. In this way, the two functions can interact with each other and their interaction can be quantified and consequently used in other calculations, such as the construction of pertinent reaction functions.

\subsection{Hedonic functions}

Hedonic methods refer to regression models in which a product's price is related to the product's characteristics and its observed price is considered to be a function of them. In general, hedonic methods are based on the idea that a product is perceived as a bundle of characteristics and consumers just buy bundles of characteristics instead of the product itself. These methods can be used to construct a quality-adjusted price index of a service. In the light of this, Triplett [21] presents an overview on hedonic price equations. Moreover, Rosen [22] states that from a large amount of product varieties, consumer chooses without influencing prices. Therefore, consumers maximize utility and producers maximize profits. In hedonic studies it is possible to adjust the price of a service for its quality and not its quantity. Regarding the construction and study of price indices for the ADSL technology services, [23, 24] refer to corresponding work carried out.

The term "hedonic methods" is related to the "hedonic function" $f(X)$, which is used in economic measurement:

$$
P_{i}=f\left(X_{i}\right)
$$

Where $P_{i}$ is the price of a variety or a model $i$ of a product and $X_{i}$ is a vector of characteristics associated with the variety. The hedonic function is then used, for different characteristics among varieties of the product for the price index estimation.

A typical example of a hedonic function is presented below:

$$
\begin{aligned}
& \ln \left(P_{i}\right)=b_{0}+b_{1} X_{1 i}+b_{2} X_{2 i}+\ldots+b_{k} X_{k i}+u_{i} \\
& i=1,2, \ldots, N
\end{aligned}
$$

In Eq. $9 P$ is the value of the $i$-th recorded value for the price of a product with a number of $k$ characteristics that influence the observed price. This constitutes a simple example, where the product's characteristics contribute linearly to the construction of the hedonic function. In other cases of hedonic functions the included characteristics can contribute in other forms, such as logarithmic, exponential or in a sinusoidal form. Moreover, $b_{i}$ corresponds to 
the parameters that must be estimated in order for the model to be completed. Based on the estimated hedonic function, the corresponding price index is constructed in a way that reflects the change in price between two subsequent time periods. Finally, the term $u_{i}$ corresponds to the regression residuals.

\section{Diffusion-price model description}

Considering the generic diffusion equation expressed in Eq. 1, the incorporation of a decision variable, such as price leads to the following equation:

$$
\frac{d F(t)}{d t}=d \cdot F(t) \cdot[S-F(t)] \cdot x(t)
$$

In Eq. 10, $x(t)$ is the current marketing effort term, which includes the effects that are happening at time $t$ and influence the adoption rate. In order to model the impact of price, $x(t)$ is defined as follows:

$$
x(t)=1+(k \cdot \text { change at time } t \text { in price })
$$

In Eq. 11, 1 represents the natural time and $k$ measures the impact and the corresponding adjustment of price over diffusion. This is represented in the following equation:

$$
x(t)=1+\left(k \cdot \frac{d P(t)}{d t}\right)
$$

$P(t)$ corresponds to the price at time $t$, which is estimated by the means of a suitably constructed price index. Thus, the model that describes the influence of the price in the diffusion process becomes:

$$
F(t)=S \cdot \frac{1}{1+e^{-\alpha-\beta \cdot(t+k \cdot P(t))}}
$$

Equation 13 captures the cumulative diffusion by simultaneously incorporating the influence of price, depicted by the factor $k \cdot P(t)$ of the equation, along with the time variable $t$, which accumulates all the underlying mechanics that drive the diffusion process.

As far as the price is concerned, the construction of a price index methodology based on hedonic functions was presented in Section 2 and expresses the relation between the prices of different varieties of a product, in correspondence with their characteristics. However, this approach does not capture the effect of demand over the pricing policy. More specifically, by the time that new technology products are introduced, they are offered at relatively high prices. These prices usually continue to stay at this high level during the 
introduction stage and they are progressively reduced, following the technology advance progress, the production cost, the competitiveness of the market as well as the corresponding acceptance of the product, expressed as the current demand. Consequently, the level of diffusion during a time period can influence the suppliers' pricing policy for the product. This happens especially in the area of telecommunications, where products and services are affected by network externalities and network effects. Thus, the more adopters of the product the higher the utility gained. Since the consumers' perceived utility is a crucial factor for a successful product life cycle, if it is combined with a suitable pricing policy it can strengthen the expectations of acceptance in the targeted market.

Based on these considerations, the proposed model incorporates the reaction of pricing process in the change of diffusion. It is based on the same assumption as in the previous case of diffusion, although in the opposite direction.

The incorporation of the influence of diffusion in the function that describes a product's price leads to the following equation:

$$
P=f\left(t, F(t), X_{i}\right)+\varepsilon
$$

In Eq. 14, $P$ is the estimated price, which is expressed as a function of the time $t$, the diffusion level of the product at that point of time, $F(t)$, and of course the rest characteristics of the product that influence its price $\varepsilon$ stands for the estimation error. For the needs of the case studies considered in this paper the following price function is used:

$$
\ln (P(t))=e^{a * \ln (D)+b * \ln (U)+c *(t+l * F(t))+d}+\varepsilon
$$

Equation 15 is in accordance with the general form of a hedonic function, as defined in Eq. 14, and corresponds to the function that provides the smallest values of the calculated errors, among the candidate ones tested. The participating variables are $D$, to denote the Downlink speed, $U$ for the Uplink speed and $t$ as well as $F(t)$ to stand for the time variable and the cumulative diffusion at time $t$ respectively. $a, b, c, l$ and $d$ are the parameters of the model and, finally, $\varepsilon$ is the estimation error. For simplicity reasons the $\ln (P)$ is used to represent the $\ln (P(t))$, which denotes that price, among other variables, is a function of time as well. Finally, it should be noted that the $P(t)$ part in Eq. 13 corresponds to the $\ln (P)$ as introduced in Eq. 15.

In general, the choice of the hedonic model is usually based on pertinent statistical measures of accuracy, such as Mean Square Error (MSE), Mean Absolute Percentage Error (MAPE) etc. An alternative approach is to base the selection on an appropriate theoretical criterion such as the Akaike Information Criterion (AIC) [25, 26]. 


\subsection{Solution algorithm}

The solution algorithm is based on the following principle: the values of the estimated parameters for the price model are inserted into the diffusion function and its corresponding parameters are estimated. The same is done for the diffusion function, i.e. the estimated parameter values of the diffusion function are inserted into the price model in order to estimate its parameters. The algorithm is terminated when convergence is achieved and no further changes to the parameter values are observed.

The set of Eqs. 13 and 15 are solved simultaneously, in an iterative way, based on the following steps, as described in [27]:

1. Set $F(t)=0, \ln (P(t))=0$ on the right-hand side of Eqs. 13 and 15, respectively.

2. Estimate parameters $(\alpha, \beta, S, \alpha, b, c, d)$ of the two resulting equations. Call them $(\alpha, \beta, S, \alpha, b, c, d)_{0}$

3. Use $(\alpha, \beta, S, \alpha, b, c, d)_{0}$, set $F(t)=0, \ln (P(t))=0$ on the right-hand sides and evaluate $F(t), \ln (P(t))$ of Eqs. 13, 15. Call these $(F(t), \ln (P(t)))_{1}$.

4. Assign $F(t), \ln (P(t))$ to the $\mathrm{F}(\mathrm{t})$ and $\ln (\mathrm{P}(\mathrm{t}))$ on the right-hand side of Eqs. 13,15 and estimate $(\alpha, \beta, S, \alpha, b, c, d)$. Call them $(\alpha, \beta, S, \alpha, b, c, d)_{1}$.

5. Using $(\alpha, \beta, S, \alpha, b, c, d)_{1}$ and $(F(t), \ln (P(t)))_{1}$ for $\mathrm{F}(\mathrm{t})$ and $\ln (\mathrm{P}(\mathrm{t}))$ on the right-hand sides, evaluate $(\mathrm{F}(\mathrm{t}), \ln (\mathrm{P}(\mathrm{t})))$ of Eqs. 13 and 15. Call these $(F(t), \ln (P(t)))_{2}$.

6. Assign $(F(t), \ln (P(t)))_{2}$ to $\mathrm{F}(\mathrm{t})$ and $\ln (\mathrm{P}(\mathrm{t}))$ on the right-hand side of Eqs. 13,15 and estimate $(\alpha, \beta, S, \alpha, b, c, d)$. Call them $(\alpha, \beta, S, \alpha, b, c, d)_{2}$.

7. Repeat Steps 5 and 6 until no observable changes in the estimates of $(\alpha, \beta$, $S, \alpha, b, c, d)$ are found.

The procedure described above is implemented using a Genetic Algorithm (GA). A brief overview of the GA, together with the reasons leading to the adoption of this approach, is presented below.

Genetic algorithms were introduced by Holland $[28,29]$ and they are adaptive heuristic search algorithms based on the mechanisms of natural systems and natural genetics. The basic concept of GAs is designed to simulate processes in natural systems necessary for evolution, specifically those that follow the principles first laid down by Charles Darwin for the survival of the fittest. As such, they represent an intelligent exploitation of a random search within a defined search space to solve a problem. The key points to the process are reproduction, crossover and mutation, which are performed according to a given probability, just as it happens in the real world. Reproduction involves copying (reproducing) solution vectors, crossover involves swapping partial solution vectors and mutation is the process of randomly changing a cell in the string of the solution vector preventing the possibility of the algorithm being trapped. The process continues until it reaches an optimal solution to the fitness function, which is used to evaluate individuals. 
Instead of GAs, estimation of a model's parameters can be alternatively based on management judgments regarding the evolution of the market, as well as competition. However, this approach could include bias, since it may reflect personal or group opinions, based on corresponding knowledge, experience and perception. On the contrary, GAs can provide accurate estimates of a model's parameters once a minimum number of data points becomes available. This is the case of telecommunications, where the available data are usually restricted to a set of a few observations, mainly due to the rapid generation substitution. As discussed in [30], the common techniques used for estimating a model's parameters, such as the Ordinary Least Squares (OLS), the Nonlinear Least Squares (NLS) and the Maximum Likelihood Estimation (MLE), are related with bias, multi-collinearity and inefficiency, of estimations. On the contrary, GAs "constitute an appropriate method to use when searching for a real number evaluation function in an optimal solution". In addition to this, theoretical arguments regarding the ability of the GAs to efficiently produce better parameter estimates are provided in [31], which are evaluated against alternative estimating methods showing the superiority of the GAs which, under certain circumstances, are able to perform better than the alternative methods, as evident in lower Mean Squared Errors (MSE) and Mean Absolute Deviation (MAD). On the other hand, when estimations are based on other methods, they may lead to problems such as values outside the allowable range, convergence problems or bias and systematic change in parameter estimates [32]. In general, GAs are capable of producing accurate estimates in cases when there are not many data points available and the solution space becomes very rough. GAs have been used to estimate demand for high technology products and they constitute a rapidly growing area of artificial intelligence [33]. They have been also applied over a wide range of optimization problems, such as solving the flexible assembly line balancing problem [34], choosing the right set of plans for queries which minimizes the total execution time [35], or solving constrained optimization problems [36].

In the context of the present work and due to the complexity of the system and the lack of sufficient data, GAs were considered as the most appropriate approach for the estimation of the models parameters.

The general steps that a GA consists of are presented below:

1. Definition of the fitness function, for the particular optimization problem.

2. Setting crossover and mutation probabilities.

3. Random generation of an initial population $N(0)$

4. Generation of $N(t+1)$ by probabilistically selecting individuals from $N(t)$ to produce offsprings via genetic operators of crossover and mutation.

5. Computation of the fitness for each individual in the current population $N(t)$. Offsprings with values closer to the fitness function are more probable to contribute with one or more offsprings to the next generation. Offsprings that diverge from the fitness function are discarded.

6. Steps 4 and 5 are repeated usually until either a prefixed number of generations is created, or after some predefined time has elapsed. 
In the evaluated case studies, the characteristics of the GAs used are the following: ${ }^{1}$

1. Objective function: The minimization of the MSE, between observed and estimated:

$$
M S E=\frac{1}{T} \sum_{t=1}^{T}(N(t)-\hat{N}(t))
$$

where $N(t), \hat{N}(t)$ are the observed and the estimated values, respectively, across all considered time periods.

2. Initial values of parameters: They were based on the initial estimations for each equation (diffusion and price). Additionally, the algorithm was executed with random initial values, in order to ensure that the algorithm would not be trapped to a local optimum.

3. Stopping condition: The algorithm is terminated when the reduction value becomes less than $0,01 \%$ in the last 10.000 iterations.

4. The population size was set to 1.000 individuals per generation, the crossover rate to 0,9 and the mutation rate to 0,01 . The operations of crossover and mutation are not performed for every reproduction but the probability of a string to be selected for crossover is proportional to the string's fitness. Each operation is assigned a particular probability of occurrence or application. The probability of mutation is always very low, since the primary function of a mutation operator is to remove the solution from a local minimum. The probabilities are assigned based on the characteristics of the problem.

\section{Case studies}

\subsection{Data}

As already mentioned, the proposed diffusion-price model was applied for the case of the ADSL technology. More specifically, four case studies are examined, regarding Germany, Belgium, Italy and the general area of Europe, including 15 European countries. The data used depict an overview of the mutual influence of penetration and price over an area of general interest, such as the European area and over the defined markets of Germany, Belgium and Italy.

Tables 1 and 2 contain the mean values of the data used for the present evaluation of the diffusion-price model in the case of European countries, while the data for the cases of Germany, Belgium and Italy are presented in Tables 3, 4, 5, 6, 7 and 8. Tables 1, 3, 5 and 7 hold the data corresponding

\footnotetext{
${ }^{1}$ Evaluation of the methodology was based on the Palisade Evolver software, a plug-in for Microsoft Excel that implements GAs (http://www.palisade.com).
} 
Table 1 Diffusion of the ADSL in the EU15 area (Source: Eurostat)

\begin{tabular}{lc}
\hline Year & Penetration \\
\hline 2001 & 2.42 \\
2002 & 5.013 \\
2003 & 8.213 \\
2004 & 12.633 \\
2005 & 17.753 \\
\hline
\end{tabular}

Table 2 Mean values for price and bandwidth characteristics for ADSL access across the EU15 countries (Source: ADSL Providers)

\begin{tabular}{llll}
\hline Year & Price $(€)$ & Downlink $(\mathrm{Kbps})$ & Uplink $(\mathrm{Kbps})$ \\
\hline 2003 & 80.16 & 1062.35 & 185.22 \\
2004 & 44.57 & 2045.88 & 606.88 \\
2005 & 48.58 & 3591.74 & 840.91 \\
\hline
\end{tabular}

Table 3 Diffusion of the ADSL in Germany (Source: Eurostat)

Table 4 Values for price and bandwidth characteristics for ADSL access in Germany (Source: ADSL Providers)

\begin{tabular}{lc}
\hline Year & Penetration \\
\hline 2001 & 2.30 \\
2002 & 4.10 \\
2003 & 5.60 \\
2004 & 8.40 \\
2005 & 13.00 \\
\hline
\end{tabular}

\begin{tabular}{llll}
\hline Year & $\begin{array}{l}\text { Price } \\
(€)\end{array}$ & $\begin{array}{l}\text { Downlink } \\
(\text { Kbps })\end{array}$ & $\begin{array}{l}\text { Uplink } \\
(\text { Kbps })\end{array}$ \\
\hline 2003 & 30.44 & 1472.00 & 192.00 \\
2004 & 34.55 & 1917.80 & 242.40 \\
2005 & 29.33 & 2672.00 & 329.81 \\
\hline
\end{tabular}

Table 5 Diffusion of the ADSL in Belgium (Source: Eurostat)

\begin{tabular}{lc}
\hline Year & Penetration \\
\hline 2001 & 4.40 \\
2002 & 8.70 \\
2003 & 11.70 \\
2004 & 15.50 \\
2005 & 18.30 \\
\hline
\end{tabular}

Table 6 Values for price and bandwidth characteristics for ADSL access in Belgium (Source: ADSL Providers)

\begin{tabular}{llll}
\hline Year & $\begin{array}{l}\text { Price } \\
(€)\end{array}$ & $\begin{array}{l}\text { Downlink } \\
(\text { Kbps })\end{array}$ & $\begin{array}{l}\text { Uplink } \\
(\text { Kbps })\end{array}$ \\
\hline 2003 & 55.88 & 3000.00 & 182.86 \\
2004 & 37.40 & 2599.20 & 224.00 \\
2005 & 42.09 & 5534.29 & 353.14 \\
\hline
\end{tabular}

Table 7 Diffusion of the ADSL in Italy (Source: Eurostat)

\begin{tabular}{lc}
\hline Year & Penetration \\
\hline 2001 & 0.70 \\
2002 & 1.70 \\
2003 & 4.10 \\
2004 & 8.10 \\
2005 & 11.90 \\
\hline
\end{tabular}


Table 8 Values for price and bandwidth characteristics for ADSL access in Italy (Source: ADSL Providers)

\begin{tabular}{llcr}
\hline Year & $\begin{array}{l}\text { Price } \\
(€)\end{array}$ & $\begin{array}{l}\text { Downlink } \\
(\mathrm{Kbps})\end{array}$ & $\begin{array}{r}\text { Uplink } \\
(\mathrm{Kbps})\end{array}$ \\
\hline 2003 & 57.09 & 484.60 & 147.20 \\
2004 & 22.36 & 3030.00 & 2131.00 \\
2005 & 22.17 & 4391.27 & 3006.55 \\
\hline
\end{tabular}

to the mean values of the ADSL penetration and price characteristics across the EU15 countries Germany, Belgium and Italy, respectively. In addition, Tables 2, 4, 6 and 8 show the mean values for price and bandwidth characteristics of the ADSL access for each of the aforementioned areas, correspondingly. The data for both diffusion and price were collected and evaluated on a quarter basis, however, they are presented as each year's mean values for space saving reasons. Penetration is defined and presented as the percentage of the adopters over the total market population of individuals.

The full dataset for ADSL prices and bandwidth characteristics, which is available in the Appendix, was used to perform the initial estimation of the price index. In order to estimate the impact of price on diffusion the CeterisParibus principle was adopted, according to which the Downlink and Uplink values of an ADSL connection were kept constant, whereas the time variable was the only variable that was allowed to change.

\subsection{Results}

The evaluation procedure consists of three steps:

- During the first step, the model's parameters are estimated based on Nonlinear Least Squares (NLS), for both the diffusion and the price functions. The models at this stage do not include the effect of price and diffusion and they are used to provide initial estimates of the processes (diffusion and price). The models' parameters are estimated separately.

- The estimates are then inserted into the new formulations of the models, described by Eqs. 13 and 15, in order to provide the estimates of the parameters that capture the simultaneous cause-effect relationship between diffusion and price (step two).

- The estimates from step two are then inserted again into the model's formulation (final, third step) and the final estimates of all participating parameters are then derived.

The last two steps of the solution algorithm are based on GAs and they are also called iterations, since they correspond to different groups of GA repetitions. The iterations are named as Iteration 1 and Iteration 2, respectively. Iteration 2 is also called "Converged", since it describes the convergence step of the algorithm. Each iteration corresponds to repetition of steps 5 and 6 of the solution algorithm, until no changes to the estimates are observed. The values estimated during Iteration 1 , do not have any special physical meaning but they 


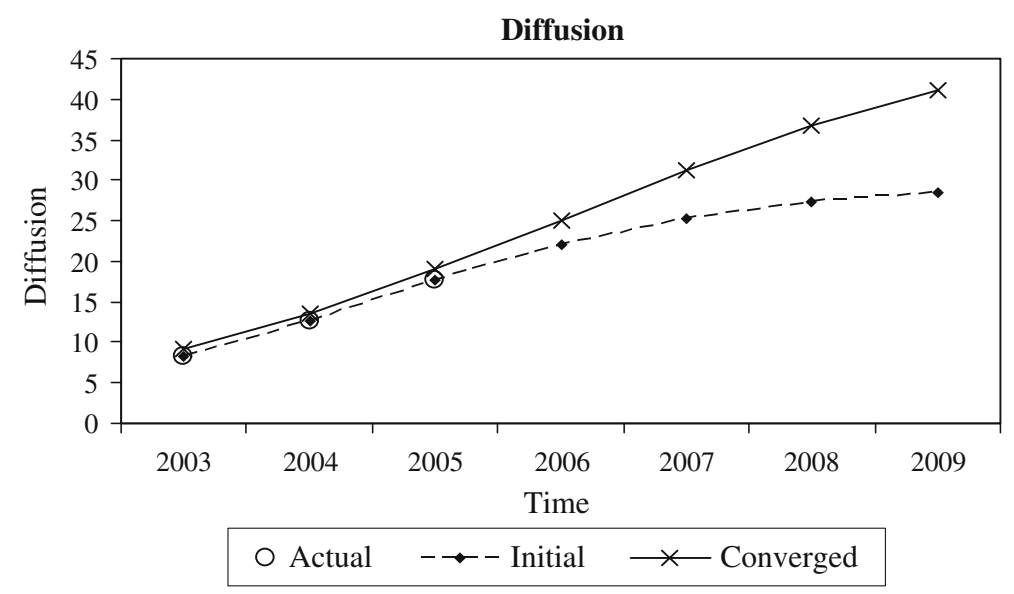

Fig. 1 Influence of price over diffusion of the ADSL technology in Europe

represent the intermediate step towards convergence, since new parameters and variables are introduced into the initial model.

Based on the above, during each iteration the GAs have created a population size of 1.000 units at each step, performing a number of about 500,000 repetitions. The procedure is terminated when no observable change is recorded during the last 10,000 repetitions.

The results obtained according to the aforementioned evaluation procedure are presented in this section. This contains the numerical results for the case of EU15 countries, which are graphically depicted in Figs. 1 and 2. The results correspond to the cases of Germany, Belgium and Italy and they are depicted in Tables 10-12 and Figs. 3, 4 and 5, respectively. In these figures, the "Converged" index corresponds to the results of Iteration 2, shown in Tables 9,

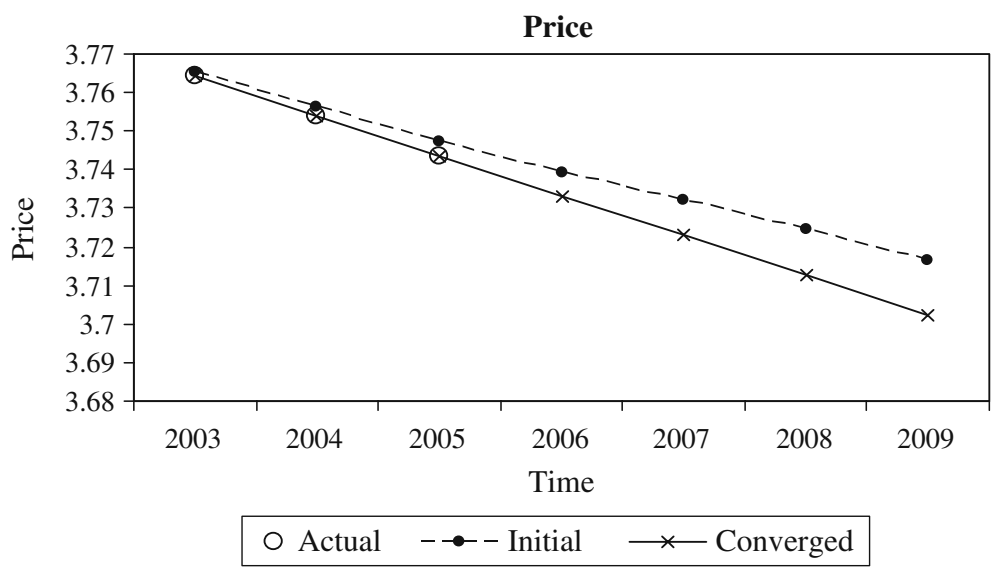

Fig. 2 Influence of diffusion over the expected pricing schema in Europe 


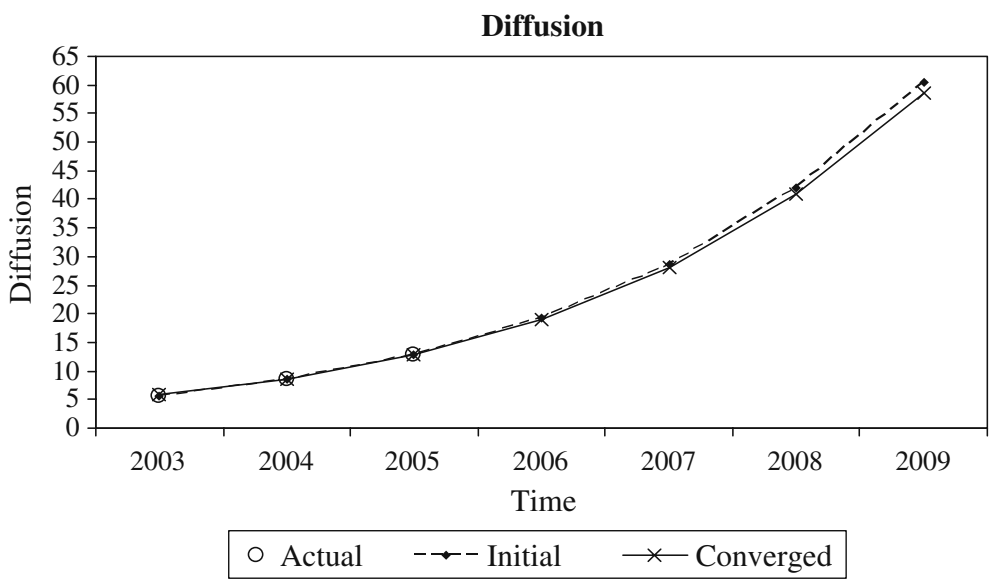

Fig. 3 Influence of price over diffusion of the ADSL technology in Germany

10,11 and 12 . The convergence of the solution algorithm described above was achieved at the second iteration.

The values of $k$ and $l$, as calculated during Iteration 2, represent the finally estimated proportionality of influence. More specifically, in the case of EU15 counties, $k$ indicates that a reduction in price contributes to an increment in diffusion by a factor of 0,6153 . The minus sign of $k$ has the meaning that there is a converse proportionality between diffusion and price, as expected. The same evidence holds for $l$, the factor of influence of diffusion over price. It is interesting to point out that, as intuitively expected, the influence of price over demand is quite higher than the opposite direction. It is obvious that by setting

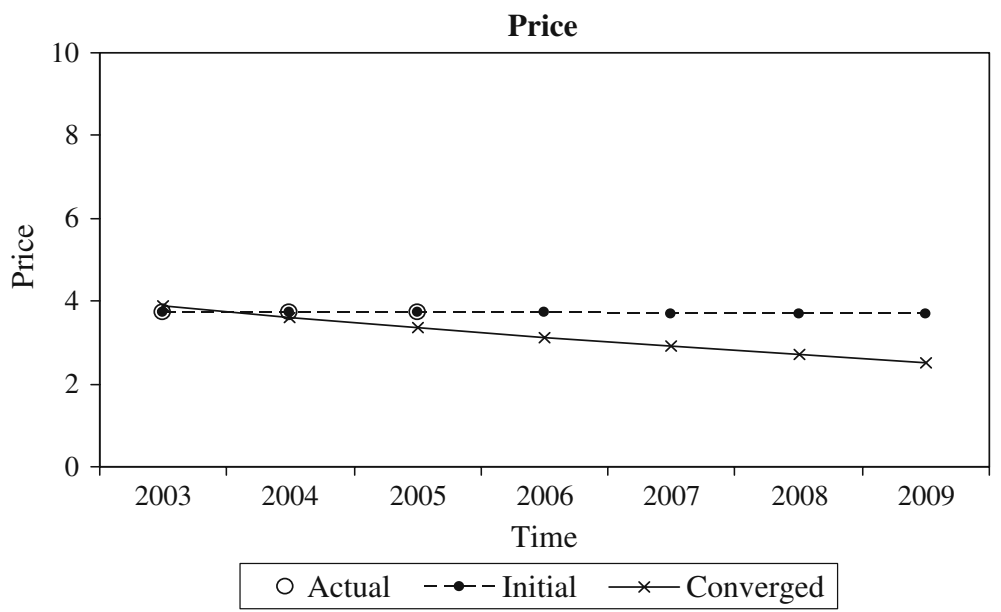

Fig. 4 Influence of diffusion over the expected pricing schema in Germany 


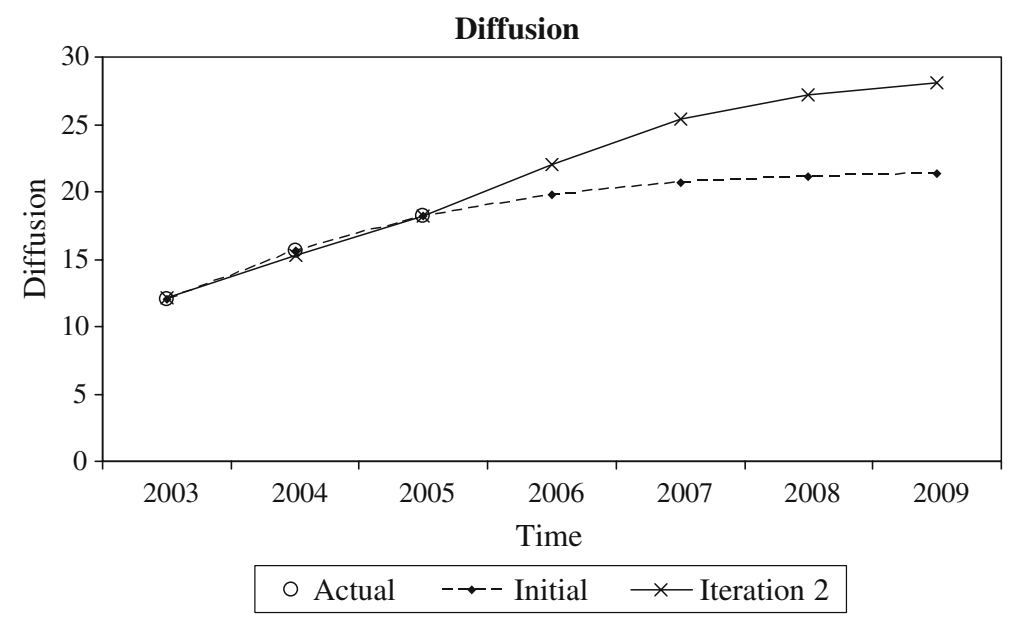

Fig. 5 Influence of price over diffusion of the ADSL technology in Belgium

Table 9 Estimation results of the diffusion-price model for EU15

Table 10 Estimation results of the diffusion-price model for Germany

\begin{tabular}{llcll}
\hline & \multicolumn{2}{l}{ Diffusion } & \multicolumn{2}{l}{ Price } \\
\hline Initial estimates & $S$ & 29.62 & $a$ & -0.068 \\
& $\alpha$ & -3.003 & $b$ & -0.014 \\
& $\beta$ & 0.68 & $c$ & -0.003 \\
Iteration 1 & $S$ & 29.62 & $d$ & 1.964 \\
& $\alpha$ & -3.003 & $a$ & -3.472 \\
& $\beta$ & 0.68 & $c$ & -0.087 \\
& & & $d$ & -0.294 \\
Iteration 2 & $k$ & -0.67 & $l$ & -1.007 \\
& $S$ & 50.18 & $a$ & -0.05 \\
& $\alpha$ & -1.824 & $b$ & -0.005 \\
& $\beta$ & 0.499 & $c$ & -0.133 \\
& & & $d$ & 1.908 \\
& $k$ & -0.615 & $l$ & -0.952 \\
\hline
\end{tabular}

\begin{tabular}{llclr}
\hline & \multicolumn{2}{l}{ Diffusion } & \multicolumn{2}{l}{ Price } \\
\hline Initial estimates & $S$ & 369.26 & $a$ & -0.068 \\
& $\alpha$ & -5.424 & $b$ & -0.014 \\
& $\beta$ & 0.421 & $c$ & -0.003 \\
Iteration 2 & & & $d$ & 1.964 \\
& $S$ & 404.98 & $a$ & -0.988 \\
& $\alpha$ & -5.459 & $b$ & $1 \mathrm{E}-300$ \\
& $\beta$ & 0.409 & $c$ & -0.072 \\
& & & $d$ & 9.505 \\
& $k$ & -2.280 & $l$ & -0.033 \\
\hline
\end{tabular}


Table 11 Estimation results of the diffusion-price model for Belgium

\begin{tabular}{llclr}
\hline & \multicolumn{2}{l}{ Diffusion } & \multicolumn{2}{l}{ Price } \\
\hline Initial estimates & $S$ & 21.49 & $a$ & -0.068 \\
& $\alpha$ & -1.986 & $b$ & -0.014 \\
& $\beta$ & 0.739 & $c$ & -0.003 \\
Iteration 1 & $S$ & 29 & $d$ & 1.964 \\
& $\alpha$ & -0.009 & $a$ & -0.375 \\
& $\beta$ & 7.086 & $c$ & -0.722 \\
& & & $c$ & -0.863 \\
Iteration 2 & $k$ & -0.214 & $l$ & -0.632 \\
& $S$ & 29.04 & $a$ & $1 \mathrm{E}-300$ \\
& $\alpha$ & -2.105 & $b$ & -0.004 \\
& $\beta$ & 0.623 & $c$ & -0.158 \\
& & & $d$ & 1.532 \\
& $k$ & -0.019 & $l$ & -0.006 \\
\hline
\end{tabular}

a lower price demand increases, but the vice versa can be the case only for the amount that makes the marginal cost of production equal the marginal profit.

As shown in Figs. 1, 3, 5 and 7, the evaluated results reveal that the saturation level of demand $(S)$ is expected to reach a substantially higher level than initially estimated, if the interaction with the corresponding pricing policy is considered. Thus, for a given set of Downlink and Uplink connection characteristics, the pricing path declines over time and at the same time diffusion increases more rapidly than calculated by using a diffusion model.

As far as Germany is concerned Figs. 3 and 4 indicate that an increment of the demand influences in a bigger degree the fall of price, than the other way round. This is explained if someone tends to think that Germany is a developed and advanced technologically country. Under these conditions a potential consumer would easily adopt any new technology or telecommunication service. Moreover, ADSL was adopted in Germany before 2003, where the telecommunications infrastructures were adequate to support the new service. As a consequence, in such a market where the ADSL has reached a mature level, the price of the technology tends to decrease over the years. In such a

Table 12 Estimation results of the diffusion-price model for Italy

\begin{tabular}{lllll}
\hline & \multicolumn{2}{l}{ Diffusion } & Price & \\
\hline Initial estimates & $S$ & 15.81 & $a$ & -0.068 \\
& $\alpha$ & -4.262 & $b$ & -0.014 \\
& $\beta$ & 1.076 & $c$ & -0.003 \\
Iteration 1 & & & $d$ & 1.964 \\
& $S$ & 51.62 & $a$ & -3.472 \\
& $\alpha$ & -9.823 & $b$ & -0.087 \\
& $\beta$ & $-1 \mathrm{E}-300$ & $c$ & -0.294 \\
Iteration 2 & & & $d$ & 1.007 \\
& $k$ & -9.9889 & $l$ & -1.967 \\
& $S$ & 62.670 & $a$ & -0.05 \\
& $\alpha$ & -4.135 & $b$ & -0.005 \\
& $\beta$ & 0.541 & $c$ & -0.133 \\
& & & $d$ & 1.908 \\
& $k$ & -1.466 & $l$ & -0.952 \\
\hline
\end{tabular}




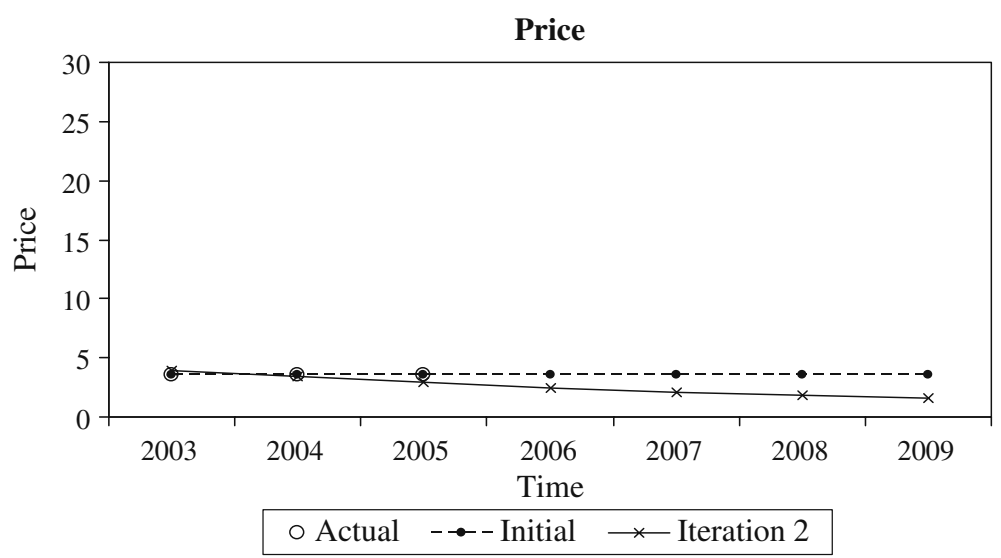

Fig. 6 Influence of diffusion over the expected pricing schema in Belgium

technologically advanced country, potential adopters may not be significantly influenced by a possible increment of the product price. Consequently and according to the aforementioned diffusion-price model an increment of the price influences the reduction of the demand than the other way round.

Regarding the case study of Belgium and Italy, Figs. 5, 6, 7 and 8 show that a possible reduction of the price involves a higher influence on the increment of the demand than the influence of a potential change of the ADSL demand to price. Potential buyers are significantly influenced by the price, which plays a critical role in order to adopt any technological innovation. More specifically, in Italy the ADSL technology was in the early years of its adoption and that's why price is not significantly reduced over the years. An important decrement would happened if the ADSL market has reached the saturation level and was ready to adopt a new generation of the ADSL technology. Under

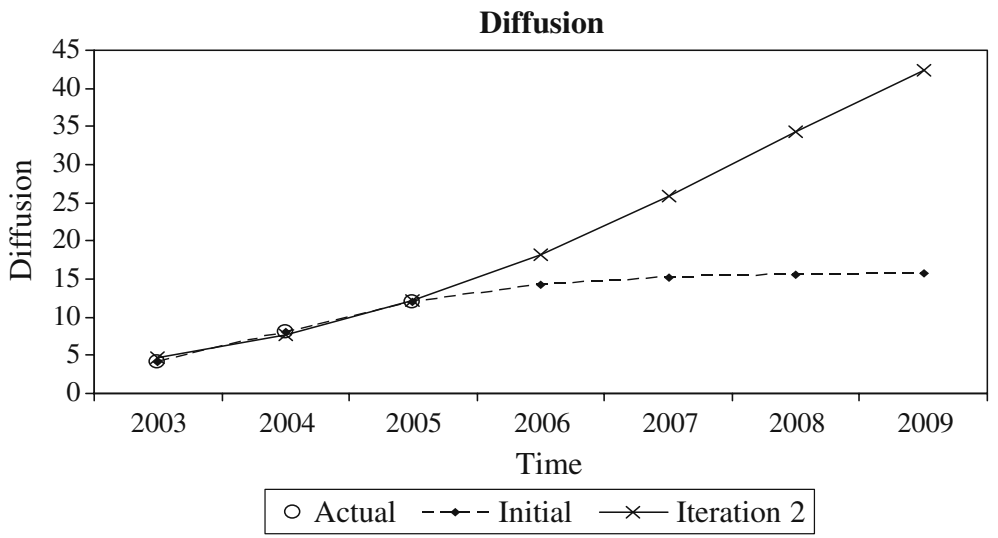

Fig. 7 Influence of price over diffusion of the ADSL technology in Italy 


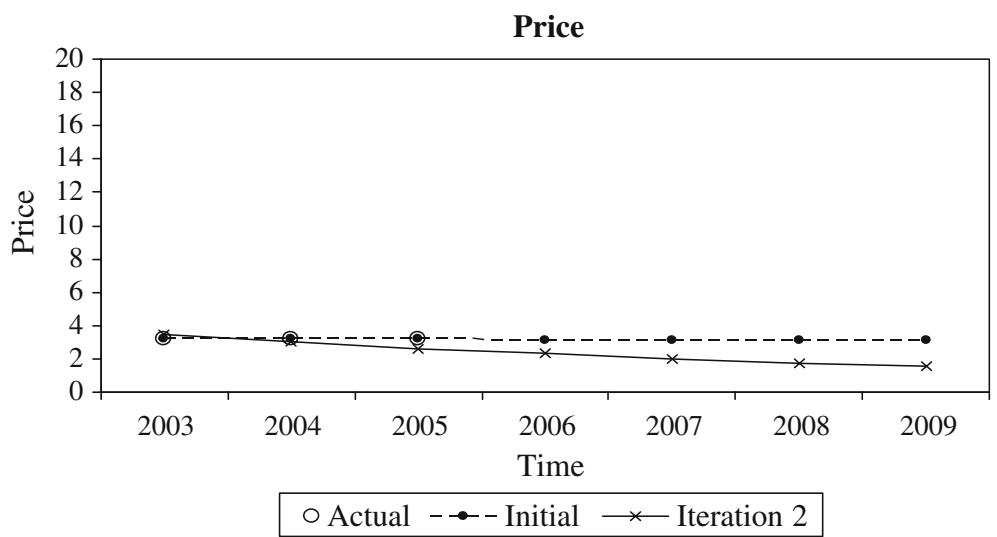

Fig. 8 Influence of diffusion over the expected pricing schema in Italy

these conditions the price would be reduced in order potential buyers to be motivated to adopt the ADSL technology than any expensive innovation of a new generation technology. Indeed, in the current case study price is a crucial factor, which influences considerably the demand of the ADSL in the countries examined.

It is interesting to note that the evaluation results can be verified by real pricing values. More specifically, in the case of Germany the initial price for year 2003 for a broadband connection was at $30.44 €$, while the current price is $24 €$ on average [37]. The evaluation of the model estimated a decrease in price of about 1 unit (from 4 to about 3 ) which corresponds to a price of $22.83 €$. Regarding Belgium, the price of $55.88 €$ in year 2003 has become $30 €$ on average [38], which is also in accordance with the model estimation indicating a price reduction of about $66 \%$. Finally, in Italy a broadband connection is currently offered at a price of about $20 €[39,40]$ which is also in accordance with our findings.

The above results verify the main contribution of the methodology, which is the examination of the reaction functions of demand and price that can lead to the estimation of the changes that are expected to happen in both of the participating quantities. Thus, the diffusion-price model could serve as a helpful tool towards the construction of a corresponding business plan, providing indications of the pricing policy that should be followed.

\section{Conclusions and future work}

In this paper, a "diffusion-price" model is introduced, into the context of a corresponding methodology, in order to incorporate the effect of a product's price over its penetration rate and vice-versa. The developed approach extends the classical diffusion theory where the majority of diffusion models consider the elapsed time since the introduction of a product as the only diffusion 
variable. However, they cannot always succeed in capturing the influence of the parameters that drive the adoption process and its characteristics, such as the product's saturation level. For this reason, the proposed methodology makes an important contribution as it provides a tool for estimating the expected price and demand elasticity at the same time, and the way they both interact.

Moreover, in the context of this work price participates not merely as recorded values but as an output of a methodologically derived process, based on price indices and hedonic functions.

Future directions for research include the incorporation of other substantially influential decision variables, such as marketing force and their consequent affection over the demand process. This will lead to the development of a proper methodological framework which will be useful for describing the diffusion process of high technology products, as influenced by the marketing variables. Moreover, incorporation of stochastic terms into the corresponding models will provide a set of possible situations of the process, at each point of time. Obviously, no matter how sophisticated a deterministic model can be, it cannot include all the factors that possibly affect the process and since many of the external parameters are random by their nature, they cannot be accurately estimated and used for forecasting purposes. Randomness can be introduced by assuming that, either the parameters of an aggregate diffusion model follow a stationary stochastic process [41], or that the future remaining growth of the underlying process is not known with certainty but is modeled using an appropriate stochastic process by an Ito's stochastic differential equation, taking into account the internal and/or external fluctuations [42].

\section{Appendix}

Table 13 Full ADSL price dataset

\begin{tabular}{llccc}
\hline Year & Country & Price $(€)$ & Downlink $(\mathrm{Kbps})$ & Uplink $(\mathrm{Kbps})$ \\
\hline 2003 & Belgium & 55.88 & 3000.00 & 182.86 \\
& France & 56.57 & 749.71 & 109.71 \\
& Germany & 30.44 & 1472.00 & 192.00 \\
& Italy & 57.09 & 484.60 & 147.20 \\
Netherlands & 58.32 & 853.33 & 149.33 \\
& Spain & 92.47 & 930.67 & 185.33 \\
& Switzerland & 155.63 & 902.74 & 322.53 \\
& UK & 132.08 & 835.33 & 250.00 \\
& Greece & 83.00 & 332.80 & 128.00 \\
& Austria & 45.32 & 1186.91 & 215.27 \\
& Belgium & 37.40 & 2599.20 & 224.00 \\
& Denmark & 58.57 & 1467.73 & 302.93 \\
& Finland & 50.69 & 1996.80 & 512.00 \\
& France & 28.93 & 1896.44 & 245.33 \\
& Germany & 34.55 & 1917.80 & 242.40 \\
& Iceland & 40.18 & 2048.00 & 512.00 \\
& Italy & 3030.00 & 2131.00 \\
& Netherlands & 45.36 & 2832.80 & 568.00 \\
Norway & 41.52 & 1594.44 & 550.81 \\
\hline
\end{tabular}


Table 13 (continued)

\begin{tabular}{llclr}
\hline Year & Country & Price $(€)$ & Downlink $($ Kbps $)$ & Uplink $($ Kbps $)$ \\
\hline \multirow{6}{*}{2005} & Spain & 87.79 & 1345.78 & 258.67 \\
& Sweden & 38.45 & 3978.56 & 2252.89 \\
& Switzerland & 49.42 & 1500.00 & 250.00 \\
& UK & 43.52 & 1247.81 & 231.06 \\
& Austria & 51.18 & 1808.57 & 1332.57 \\
& Belgium & 42.09 & 5534.29 & 353.14 \\
Denmark & 44.09 & 1676.00 & 286.00 \\
& Finland & 36.62 & 3245.54 & 600.65 \\
& France & 30.65 & 7469.87 & 939.73 \\
Germany & 29.33 & 2672.00 & 329.81 \\
Iceland & 88.27 & 3072.00 & 512.00 \\
Italy & 22.17 & 4391.27 & 3006.55 \\
& Netherlands & 46.10 & 4668.82 & 681.36 \\
Norway & 68.11 & 5323.41 & 649.59 \\
Spain & 79.24 & 1613.60 & 245.60 \\
& Sweden & 37.36 & 7788.00 & 2820.89 \\
Switzerland & 49.32 & 1500.00 & 250.00 \\
UK & 44.14 & 1792.00 & 291.52 \\
Greece & 60.06 & 1320.73 & 314.18 \\
\hline
\end{tabular}

\section{References}

1. Bass, F. M. (1969). A new product growth model for consumer durables. Management Science, 15, 215-227.

2. Mansfield, E. (1961). Technical change and the rate of imitation. Econometrica, 29, 741-766.

3. Rogers, E. M. (1962). Diffusion of innovations. New York: The Free Press.

4. Teng, J. T. C., Grover, V., \& Guttler, W. (2002). Information technology innovations: General diffusion patterns and its relationships to innovation characteristics. IEEE Transactions on Engineering Management, 49, 13-27.

5. Linton, J. D. (2002). Forecasting the market diffusion of disruptive and discontinuous innovation. IEEE Transactions on Engineering Management, 49, 365-374.

6. Meade, N., \& Islam, T. (2006). Modelling and forecasting the diffusion of innovationA 25-year review. International Journal of Forecasting, 22, 519-545.

7. Fildes, R., \& Kumar, P. (2002). Telecommunications demand forecasting—a review. International Journal of Forecasting, 18, 489-522.

8. Robinson, B., \& Lakhani, C. (1975). Dynamic price models for new-product planning. Management Science Series B-Application, 21, 1113-1122.

9. Martin, B. W., \& Seung, J. S. (2004). Internet interconnection economic model and its analysis: Peering and settlement. Netnomics, 6, 43-57.

10. Mandjes, M. (2004). Pricing strategies and service differentiation. Netnomics, 6, 59-81.

11. Deligiorgi, C., Vavoulas, A., Michalakelis, C., \& Varoutas, D. (2007). On the construction of price index and the definition of factors affecting tariffs of ADSL connections across Europe. Netnomics, 8, 171-183.

12. Karamti, C., \& Grzybowski, L. (2010). Hedonic study on mobile telephony market in France: pricing-quality strategies. Netnomics, 11, 1-35.

13. Le Cadre, H., Bouhtou, M., \& Tuffin, B. (2009). Consumers' preference modeling to price bundle offers in the telecommunications industry: A game with competition among operators. Netnomics, 10, 171-208.

14. Michalakelis, C., Dede, G., Varoutas, D., \& Sphicopoulos, T. (2005). Impact of cross-national diffusion process in telecommunications demand forecasting. In NAEC 2005 (pp. 632-640). Garda, Italy.

15. Fisher, J. C., \& Pry, R. H. (1971). A simple substitution model of technological change. Technological Forecasting and Social Change, 3, 75-88. 
16. Bewley, R., \& Fiebig, D. G. (1988). A flexible logistic growth-model with applications in telecommunications. International Journal of Forecasting, 4, 177-192.

17. Rai, L. P. (1999). Appropriate models for technology substitution. Journal of Scientific \& Industrial Research, 58, 14-18.

18. Michalakelis, C., Varoutas, D., \& Sphicopoulos, T. (2008). Diffusion models of mobile telephony in Greece. Telecommunications Policy, 32, 234-245.

19. Bass, F. M., Krishnan, T. V., \& Jain, D. C. (1994). Why the bass model fits without decision variables. Marketing Science, 13, 203-223.

20. Heeler, R. M., \& Hustad, T. P. (1980). Problems in predicting new product growth for consumer durables. Management Science, 26, 1007-1020.

21. Triplett, J. E. (2004). Hand book on hedonic indexes and quality adjustments in price indexes. OECD Publishing.

22. Rosen, S. (1974). Hedonic prices and implicit markets: Product differentiation in pure competition. Journal of Political Economy, 92, 34-55.

23. Deligiorgi, N., Vavoulas, A., Michalakelis, C., Varoutas, D., \& Sphicopoulos, T. (2006). Factors and characteristics that affect ADSL tariffs. In 5th conference of telecommunication, media and internet techno-economics (CTTE2006), 8-9 June, Athens, Greece.

24. Deligiorgi, N., Vavoulas, A., Michalakelis, C., Varoutas, D., \& Sphicopoulos, T. (2006). Nonparametric estimation of a hedonic price index for ADSL connections in the European market using the Akaike information criterion. In Networking and e-commerce conference, NAEC 2006. Lake Garda, Italy.

25. Simonoff, J. S., \& Tsai, C. L. (1999). Semiparametric and additive model selection using an improved Akaike information criterion. Journal of Computational and Graphical Statistics, 8 , 22-40.

26. Naik, P. A., \& Tsai, C. L. (2001). Single-index model selections. Biometrika, 88, 821-832.

27. Kumar, V., \& Krishnan, T. V. (2002). Multinational diffusion models: An alternative framework. Marketing Science, 21, 318-330.

28. Goldberg, D. E. (1989). Genetic algorithms in search, optimization, and machine learning. Reading, Mass: Addison-Wesley.

29. Holland, J. (1975). Adaption in natural and artificial systems. Ann Arbor, Michigan: University of Michigan Press.

30. Wang, F.-K., \& Chang, K.-K. (2009). Modified diffusion model with multiple products using a hybrid GA approach. Expert Systems with Applications, 36, 12613-12620.

31. Venkatesan, R., Krishnan, T., \& Kumar, V. (2004). Evolutionary estimation of macro-level diffusion models using genetic algorithms: An alternative to nonlinear least squares. Marketing Science, 23, 451-464.

32. Van den Bulte, C., \& Lilien, G. L. (1997). Bias and systematic change in the parameter estimates of macro-level diffusion models. Marketing Science, 16, 338-353.

33. Venkatesan, R., \& Kumar, V. (2002). A genetic algorithms approach to growth phase forecasting of wireless subscribers. International Journal of Forecasting, 18, 625-646.

34. Guo, Z. X., Wong, W. K., Leung, S. Y. S., Fan, J. T., \& Chan, S. F. (2008). A genetic-algorithmbased optimization model for solving the flexible assembly line balancing problem with work sharing and workstation revisiting. IEEE Transactions on Systems Man and Cybernetics Part C-Applications and Reviews, 38, 218-228.

35. Bayir, M. A., Toroslu, I. H., \& Cosar, A. (2007). Genetic algorithm for the multiple-query optimization problem. IEEE Transactions on Systems Man and Cybernetics Part C-Applications and Reviews, 37, 147-153.

36. Wang, Y., Cai, Z. X., Guo, G. Q., \& Zhou, Y. R. (2007). Multiobjective optimization and hybrid evolutionary algorithm to solve constrained optimization problems. IEEE Transactions on Systems Man and Cybernetics Part B-Cybernetics, 37, 560-575.

37. http://www.tempobox.de/en/index.html.

38. http://www.base.be/fr/internet/home-internet/.

39. http://www.alice.it/.

40. http://www.fastweb.it/offerte/?WT.mc_id=fastwebmenu1.

41. Karmeshu \& Pathria, R. K. (1980). Stochastic-evolution of a non-linear model of diffusion of information. Journal of Mathematical Sociology, 7, 59-71.

42. Meade, N. (1989). Technological substitution - a framework of stochastic-models. Technological Forecasting and Social Change, 36, 389-400. 